Psychotherapeut 2021 · 66:398-405

https://doi.org/10.1007/s00278-021-00532-3

Angenommen: 20. Juli 2021

Online publiziert: 25. August 2021

(c) Springer Medizin Verlag $\mathrm{GmbH}$, ein Teil von Springer Nature 2021

\section{Videobasierte Behandlungen in der psychodynamischen Psychotherapie in Zeiten der COVID-19-Pandemie}

\author{
Interviewstudie mit Psychotherapeut*innen und \\ Patient*innen
}

\section{In diesem Beitrag}

- Material und Methoden Stichprobe - Datenerhebung - Datenanalyse

- Ergebnisse

I. Kernkategorie • II. Kernkategorie • III. Kernkategorie

- Diskussion

Interpretation der Ergebnisse und zukünftig zu falsifizierende Erkenntnisse • Methodische Limitationen und Forschungsausblick

\section{Zusatzmaterial online}

Die Online-Version dieses Beitrags (https:// doi.org/10.1007/s00278-021-00532-3) enthält Tabellen für Hintergrundinformationen.

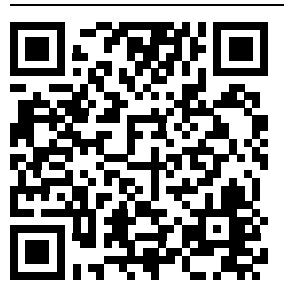

QR-Code scannen \& Beitrag online lesen

Zusammenfassung

Hintergrund: Im Zuge der durch die "coronavirus disease 2019" (COVID-19) ausgelösten Pandemie haben videobasierte Therapien (VBT) eine Trendwende erfahren. Es lässt sich vermuten, dass der abrupte Wechsel von der traditionellen Psychotherapie auf das Video-Setting (VS) einen Einfluss auf die therapeutische Beziehung und damit auf den therapeutischen Prozess ausübt. Im Rahmen dieser Studie wird untersucht, wie der Wechsel von der traditionellen Behandlung zur videobasierten Behandlung (und wieder zurück) während der COVID-19-Pandemie im Hinblick auf die therapeutische Beziehung und den therapeutischen Prozess von Patient*innen und Therapeut*innen erlebt wurde.

Methodik: Gruppenspezifische, halbstrukturierte Interviews mit approbierten Therapeut*innen $(n=8)$, Therapeut*innen in Ausbildung $(n=6)$ und Patient*innen $(n=9)$.

Ergebnisse: Die Mehrheit der Teilnehmer*innen, sowohl Therapeut*innen als auch Patient*innen, hat die therapeutische Situation im VS als durchlässiger und weniger berechenbar erlebt. Obwohl das VS zum Erhalt der therapeutischen Beziehung beiträgt, wird die Interaktion als flacher und weniger gerichtet beschrieben. Weiterhin zeigte sich, dass jeder Setting-Wechsel mit einem Habituationsprozess verbunden ist. Die therapeutische Arbeit kann dadurch erst nach einigen Sitzungen mit der höchst möglichen Intensität innerhalb des jeweiligen Settings durchgeführt werden.

Diskussion: In der VBT kann es durch den Erhalt der Beziehung zu einer Stärkung in der therapeutischen Allianz kommen. Obwohl das VS mit Unsicherheiten verbunden ist, konnten schambesetzte Themen leichter angesprochen werden. Besonders Angstpatient*innen konnten sich nach einer universell beobachtbaren Habituationsphase auf einen intensiveren therapeutischen Prozess einlassen.

\title{
Schlüsselwörter
}

Setting-Wechsel · Therapeutischer Prozess · Therapeut-Patient-Beziehung · Patient-TherapeutInteraktion · Online-Therapie

Im Zuge der durch die „coronavirus disease 2019" (COVID-19) ausgelösten Pandemie wurden Psychotherapeut*innen in Deutschland mit der Notwendigkeit, videobasierte Therapien (VBT) durchzuführen, konfrontiert. Die Richtlinien der kassenärztlichen Vereinigungen (KV) sind hinsichtlich der Rahmenbedingungen von Therapien im Video-Setting (VS) an diese Notlage angepasst worden. Weniger Therapieausfälle waren die Folge, wodurch die therapeutische Beziehung 
aufrechterhalten werden konnte. Es besteht bisher allerdings kein fundiertes Wissen darüber, welchen Einfluss das VS auf psychodynamische Therapieprozesse in Zeiten der COVID-19-Pandemie ausübt.

\section{Einleitung}

Der Einsatz digitaler Medien im psychotherapeutischen Kontext wurde von Beginn an kontrovers diskutiert (Eichenberg 2021). Obwohl sich, basierend auf einer Umfrage der Deutschen Psychotherapeutenvereinigung (DPtV) im April 2020,73 \% der befragten Psychotherapeut*innen kurzfristig für den Wechsel ins VS entschieden haben, schätzten 59\% der Befragten die Wirksamkeit der videobasierten Behandlung, im Vergleich zum traditionellen "Face-toface"-Setting, als schlechter ein (Deutsche Psychotherapeutenvereinigung 2020). Da sich die therapeutische Interaktion im VS jedoch in vielerlei Hinsicht vom Face-toface-Setting unterscheidet, ist eine fundierte Aufklärung hinsichtlich Setting-spezifischer Besonderheiten und möglicher Risiken, v.a. im Hinblick auf die weniger erfahrenen Praktiker*innen, unerlässlich.

Verglichen mit dem angloamerikanischen Sprachraum wurden innerhalb Deutschlands bisher wenige Untersuchungen zu dieser Thematik unternommen. Scharff (2013) präsentierte bereits vor einigen Jahren umfassende Überlegungen zu den aus der Virtualisierung des TherapieSettings entstehenden Konsequenzen für psychodynamisch ausgerichtete Therapieverfahren. Entsprechende Unternehmungen, mit dem Ziel, die Behandlungstechnik der VBT innerhalb Deutschlands zu manualisieren, wurden erst mit dem Einbruch der Pandemie angestoßen (HartmannStrauss 2020). Hierbei wurden bisher jedoch kaum konkrete Hinweise zur Behandlungstechnik vorgestellt.

Obwohl die Wirksamkeit videobasierter Therapien als mit dem Face-to-faceSetting vergleichbar beschrieben wurde (Backhaus et al. 2012; Berryhill et al. 2019; Norwood et al. 2018), ist weiterhin eine allgemeine Skepsis hinsichtlich dieser Behandlungsform zu beobachten (Deutsche Psychotherapeutenvereinigung 2020; Roesler 2017). Vor allem in psychodynamischen Fachkreisen wer- den Argumente für und wider die VBT ambivalent diskutiert und zugleich, verglichen mit Unternehmungen im Bereich der Verhaltenstherapie, kaum systematisch untersucht (Backhaus et al. 2012; Berryhill et al. 2019). Es zeigte sich, dass Verhaltenstherapeut*innen signifikant positivere Einstellungen zum videobasierten Behandlungsformat aufweisen als psychodynamisch orientierte Psychotherapeut*innen (Békés und Aafjes-van Doorn 2020). Weiterhin wurde im Rahmen der Umfrage der DPtV im Frühjahr 2020 durch die befragten Psychotherapeut*innen vermehrt angegeben, dass psychotherapeutische Videositzungen für die psychodynamische Psychotherapie wenig adäquat seien (Deutsche Psychotherapeutenvereinigung 2020).

Da sich persönliche Einstellungen und Erwartungen gegenüber der VBT als mögliche Einflussgrößen hinsichtlich der Wirksamkeit der Behandlung erwiesen haben (Reese et al. 2016; Tonn et al. 2017), ist das individuelle Erleben dieses abrupten und im Rahmen der Pandemie häufig auferlegten Wechsels ins VS von zentraler Bedeutung. Es kann angenommen werden, dass der kurzfristige Setting-Wechsel einen nicht zu vernachlässigenden Einfluss auf die therapeutische Beziehung und damit auf den therapeutischen Prozess ausübt. Im VS entstehe eine lediglich vordergründige Nähe, deren therapeutische Wirksamkeit infrage zu stellen sei (Jaeger und Furck 2014; White 2020). White (2020) beschreibt die physische Präsenz als eine Kernkomponente der psychodynamischen Arbeit. Im VS können Übertragungssignale weniger gut aufgenommen werden, was eine Verletzung der Neutralität und Abstinenz mit sich bringe (Scharff 2013). Ein verringerter Transport unbewusster, nonverbaler Signale könne einen erschwerten Aufbau des therapeutischen Arbeitsbündnisses zur Folge haben, wodurch Therapieerfolge weniger wahrscheinlich würden (Hoffmann et al. 2020; Roesler 2017). Da emotionale Sicherheit stärker über nonverbale als über verbale Signale vermittelt werde, könne dieser Informationsverlust besonders für Patient*innen von Bedeutung sein, die Schwierigkeiten haben, sich auf eine vertrauensvolle therapeutische Beziehung einzulassen (Roesler 2017). Durch den Einsatz moderner Medi- en im therapeutischen Kontext sei außerdem die Gefahr hoch, dass Grenzen verletzt werden (Eichenberg 2020). Die vorliegende Arbeit beschäftigt sich mit der Fragestellung, ob und inwiefern der abrupte Wechsel vom Präsenz-Setting (PS) ins VS (und wieder zurück) mit Veränderungen innerhalb der therapeutischen Beziehung und des psychodynamischen Therapieprozesses im Zusammenhang steht.

\section{Material und Methoden}

Ausgehend von der Universitätsambulanz der Sigmund Freud PrivatUniversität Berlin wurden im Zeitraum vom 01.07.2020 bis zum 30.11.2020 approbierte Psychotherapeut*innen, Psychotherapeut*innen in Ausbildung ( $\mathrm{PiA})$ und Psychotherapiepatient*innen telefonisch befragt.

\section{Stichprobe}

Grundlage dieser Studie sind anonymisierte Daten von insgesamt 23 Proband*innen, die sich in folgende Gruppen unterteilen: 1. approbierte Psychotherapeut*innen ( $n=8$; durchschnittliches Alter $=53,93$ Jahre, Standardabweichung $[\mathrm{SD}] \pm 8,02$ Jahre; w: $n=7 ; \mathrm{m}$ : $n=1), 2$. PiA ( $n=6$; durchschnittliches Alter $=38,33$ Jahre, $S D \pm 8,01$ Jahre; $w: ~ n=5$; $\mathrm{m}: n=1), 3$. Psychotherapiepatient ${ }^{*}$ innen ( $n=9$; durchschnittliches Alter $=42,5$ Jahre, SD $\pm 10,9$ Jahre; w: $n=5 ; \mathrm{m}: n=4$ ) mit unterschiedlichen F-Diagnosen: F3x.x: $n=7$; F4x.x: $n=6$; F6x.x: $n=2$ (Zusatzmaterial online: Tab. 1 und 2 für eine genauere Stichprobenbeschreibung).

Alle Proband*innen sind volljährig, deutschsprachig und im Rahmen des deutschen Kassensystems praktizierend bzw. in psychotherapeutischer Behandlung mit psychodynamischer Ausrichtung. In die Erhebung eingeschlossene Psychotherapeut*innen $(n=14)$ konnten entweder die psychotherapeutische Approbation mit psychoanalytischer (AP; $n=2)$ und/oder tiefenpsychologischer Fachkunde (TP; $n=6$ ) vorweisen oder befanden sich in einer psychotherapeutischen Approbationsausbildung (AP: $n=0$; TP: $n=6)$. Zur Gewährleistung einer ausreichenden Erfahrung mit dem VS war außerdem die Durchführung bzw. die Inanspruchnahme von mindestens 5 Sit- 
zungen (einschließlich der Probatorik) in Einzeltherapie Einschlusskriterium. Inkludierte Therapeut*innen sollten mindestens 3 Patient*innen im VS behandelt haben.

Basierend auf 2 offenen Pilotinterviews mit einer Psychotherapeutin sowie einer Patientin, die sich zu dem Zeitpunkt in psychodynamisch ausgerichteter Psychotherapie befand, wurden im ersten Schritt entsprechend dem "theoretical sampling" (Breuer et al. 2019) relevante Fallgruppen definiert: approbierte Psychotherapeut*innen, Psychotherapeut*innen in Ausbildung und Psychotherapiepatient*innen. Der Grounded-Theory-Methodologie (Corbin und Strauss 2015; Glaser und Strauss 1967) folgend entstanden bereits zentrale Kategorien (selektive Kodes), auf deren Grundlage gruppenspezifische Interviewleitfäden erstellt wurden. Im Anschluss wurde mit 3 Proband*innen aus der jeweiligen Fallgruppe jeweils ein weiteres Interview geführt, das zur Überarbeitung der bisherigen Kategorien sowie des Interviewleitfadens führte (Zusatzmaterial online: Tab. 3).

Geprägt durch die zeitliche Begrenztheit, in die das Phänomen des abrupten Wechsels ins VS eingebettet war, wurde im weiteren Verlauf entsprechend dem "Convenience sampling" rekrutiert. Die Rekrutierung der Patient*innen erfolgte in einigen Fällen über deren Therapeut*innen. Eine Studienteilnahme dieser Therapeut*innen war nicht vorgesehen. Alternativ wurden Proband*innen über Informationsmaterial, das über fachnahe Newsletter, Onlineforen oder in psychotherapeutischen Einrichtungen bereitgestellt wurde, auf die Studie aufmerksam gemacht. Mit dem Ziel der Abbildung möglichst großer Stichprobenheterogenität wurde ein Stichprobenumfang von mindestens 6 Proband*innen/Gruppe festgelegt. Eine weitere Stichprobenziehung wurde bei Erreichen einer konsensuell festgelegten theoretischen Sättigung beendet.

Alle Studienteilnehmer*innen wurden hinsichtlich der Teilnahme umfassend aufgeklärt und erhielten im Zuge eines Onlinefragebogens zur Erhebung der soziodemografischen Daten die Möglichkeit, eine persönliche Chiffre zu erstellen. So konnte die Zuordnung zwischen Frage- bogen- und Interviewdaten anonymisiert erfolgen.

\section{Datenerhebung}

Die Erfahrungen der Studienteilnehmer*innen wurden anhand halbstrukturierter Interviews erfragt. Für die oben genannten Gruppen wurde jeweils ein*eine Interviewer*in festgelegt, der*die alle Interviews innerhalb dieser Gruppe durchführte. Alle Interviews wurden telefonisch durchgeführt und mithilfe eines externen Aufnahmegeräts aufgezeichnet und gespeichert. Die Dauer der Interviews variierte zwischen 30 und $50 \mathrm{~min}$. Im Anschluss an die Interviews wurden die Audiodateien transkribiert und in die Auswertungssoftware MAXQDA 2020 (VERBI Software 2019) überführt (ausgewählte Interviewzitate werden im Zusatzmaterial online: Tab. 4, präsentiert).

\section{Datenanalyse}

Im Rahmen der präsentierten Ergebnisse wurde der thematische Fokus des pandemiebedingten Setting-Wechsels auf die therapeutische Beziehung und den therapeutischen Prozess eingegrenzt. Die Auswertung der Daten erfolgte in Anlehnung an die Grounded-Theory-Methodologie nach Glaser und Strauss (Corbin und Strauss 2015; Glaser und Strauss 1967). Der Ablauf dieses Vorgehens beinhaltet das offene, axiale und selektive Kodieren mit dem Ziel der Formulierung eines theoretischen Modells eingeschränkter Reichweite (Breuer et al. 2019). Die Auswertungsschritte wurden parallel durch zwei Mitarbeiter*innen ausgeführt (,independent coding", Barber und Walczak 2009). In regelmäßigen Abständen wurden die Zwischenergebnisse durch eine weitere Mitarbeiterin, die vom eigentlichen Analyseprozess unabhängig war, gegengeprüft und kommentiert (,peer debriefing"; Barber und Walczak 2009). Die im Rahmen dieses Austauschs entstandenen Erkenntnisse flossen in den Prozess der Theoriebildung ein, was eine Validierung der Kodierung ermöglichte (Breuer et al. 2019; Dourdouma und Mörtl 2012).

Die an der Auswertung beteiligten Personen sind Psycholog*innen in psycho- therapeutischer Ausbildung mit psychodynamischer Ausrichtung bzw. mit Fachkunde in analytischer Psychotherapie. Die beiden Mitarbeiter*innen, die für die Kodierung zuständig waren, haben im Zuge der Pandemie selbst VBT angeboten und hierbei überwiegend positive Erfahrungen gemacht, wohingegen die Mitarbeiterin, die im Rahmen des Peer debriefing beteiligt war, zum Zeitpunkt der Auswertung keine persönliche Erfahrung mit der VBT vorweisen konnte und der Therapieform gegenüber unvoreingenommen war. Alle am Analyseprozess beteiligten Personen waren sich ihrer Subjektivität innerhalb der Datenanalyse und -interpretation bewusst. Die Prozesse des Peer debriefing, der Einsatz einer unabhängigen Kodiererin sowie die ständige Reflexion des persönlichen Einflusses auf die Studienergebnisse dienen der Sicherstellung einer möglichst umfassenden Gültigkeit der vorliegenden Ergebnisse (Breuer et al. 2019; Corbin und Strauss 2015).

\section{Ergebnisse}

Im Folgenden werden die ermittelten Metakategorien für Patient*innen $(P)$ und Therapeut*innen (mit Approbation oder $\mathrm{PiA}$; T) beschrieben. Es werden also die Inhalte vorgestellt, die sich innerhalb der jeweiligen Gruppe, entsprechend der thematischen Eingrenzung des pandemiebedingten Setting-Wechsels, auf die therapeutische Beziehung und den therapeutischen Prozess am deutlichsten herausgestellt haben. Da sich zwischen den approbierten Therapeut*innen und den PiA im Zuge der axialen Kodierung kaum systematische Unterschiede ergeben haben, werden diese in einer Gruppe zusammengefasst (T). Einzelne Abweichungen zwischen approbierten Therapeut*innen und $\mathrm{PiA}$ werden explizit hervorgehoben. Für alle Gruppen konnten bereits in der zuvor beschriebenen Phase des Theoretical sampling insgesamt 10 selektive Kodes ermittelt werden (Zusatzmaterial online: Tab. 5). Durch eine weitere Ausdifferenzierung der axialen Kodes konnten die Ergebnisse im Zuge der vertieften Analyse abduktiv zu 3 Kernkategorien verdichtet werden (Zusatzmaterial online: Tab. 4). 


\section{Kernkategorie}

Die therapeutische Situation wird im VS durchlässiger und weniger berechenbar.

\section{Therapeutischer Raum}

T. Der therapeutische Raum geht im VS verloren; dies erschwert das Zustandekommen von Resonanz und das Einnehmen der Position eines containenden Gegenübers. Verglichen mit dem PS haben die Therapeut*innen im VS nur begrenzten Einfluss auf die Gestaltung des therapeutischen Rahmens. Hierdurch wird das im traditionellen Setting vorhandene Gefühl von Sicherheit gestört. Besonders irritierend erleben dies Patient ${ }^{*}$ innen, denen es ohnehin schwerfällt, Vertrauen aufzubauen.

P. Im VS ist es wichtig, einen ruhigen und geschützten Raum zur Verfügung zu haben. Bei einer Neigung zu sozialer Vermeidung wird das VS als angenehmer und sicherer wahrgenommen als der Besuch in der Praxis des*der Therapeut*in.

\section{Organisation des Video-Settings}

T. Obwohl dem*der Patient*in innerhalb des VS eine vertraute Umgebung, ausgehend von der Praxis des*der Therapeut*in als Behandlungsort, präsentiert werden kann, führten einige Therapeut*innen Sitzungen im VS auch in ihrem Zuhause durch. Dies kann einerseits zur Auflockerung der therapeutischen Atmosphäre beitragen, andererseits auch mit der Empfindung von Stress einhergehen. Tiefenpsychologisch orientierte Psychotherapeut*innen bevorzugen den Blickkontakt im VS, während analytisch arbeitende Therapeut*innen ein Abschalten der Kamera bevorzugen.

P. Bei der Organisation des Settings wird darauf geachtet, immer am selben Platz zu sitzen und einen neutralen Hintergrund zu schaffen. Mitbewohner*innen und Angehörige werden gebeten, dem Raum, in dem die Therapie durchgeführt wird, fernzubleiben. Darüber hinaus spielt es eine Rolle, in welchem Raum sich der* die Therapeut*in befindet. Sind im Hintergrund die gewohnten Praxisräume erkennbar, wird dies als beruhigend erlebt.

\section{Unterschiede im Setting}

T. Das VS unterscheidet sich am deutlichsten vom PS hinsichtlich der begrenzten Möglichkeit, auf nonverbaler Ebene zu kommunizieren. Im Vergleich zum PS wird der Kontakt im VS als weniger persönlich empfunden. Die Phänomenologie der vorliegenden Erkrankung verändert sich außerdem im VS.

P. Im VS besteht im Vergleich zum PS mehr Distanz zum*zur Therapeut*in. Er*sie ist weniger greifbar und wird als weniger real empfunden. Die eingeschränkte oder fehlende Wahrnehmbarkeit von Körpersprache, Mimik und Olfaktorik wird als Mangel erlebt. Weiterhin werden sowohl der Weg zur Therapie als auch der Rückweg von der Therapie im PS als Ressource und Wirkfaktor erlebt, was im VS entfällt. Das Wegfallen des Wegs in die und von der therapeutischen Praxis empfinden Patient*innen mit Angsterkrankungen jedoch als angenehm.

\section{Technische Voraussetzungen}

T. Technische Störungen verhindern den therapeutischen Prozess. Zur Schaffung der notwendigen technischen Grundvoraussetzungen im VS müssen Therapeut*innen vergleichsweise mehr Zeit für die einzelnen therapeutischen Sitzungen aufwenden. Die Nutzung zertifizierter Programme wirkt vertrauenserweckend, sowohl auf Therapeut*innen als auch auf Patient*innen.

P. Die Technik stellt bei Vorkenntnissen mit Videotelefonie kein ernsthaftes Problem dar. Da ein reibungsloser Ablauf der Sitzungen als sehr wichtig wahrgenommen wird, lösen technische Störungen innerhalb der Sitzungen negative Gefühle aus. Meistens funktioniert die verwendete Technik jedoch ausreichend gut.

\section{Kernkategorie}

Obwohl das VS zum Erhalt der therapeutischen Beziehung beiträgt, wird die Interaktion flacher und weniger gerichtet. Gleichzeitig konnte die intersubjektive Ebene verstärkt thematisiert werden.

\section{Therapeutische Beziehung}

T. Die therapeutische Beziehung kann im VS aufrechterhalten werden, obwohl der persönliche Kontakt und die physische Nähe hier fehlen. Auffällig ist eine Verschiebung der empfundenen Kontrolle im VS in Richtung der Patient*innen. Die therapeutischen Gespräche ähneln dem Austausch mit Angehörigen und Freunden. Je länger vor dem Setting-Wechsel Kontakt bestanden hat, desto besser entwickelt sich der therapeutische Prozess. Ein begünstigender Faktor für den Einsatz der VBT ist nach Einschätzung der PiA eine "gemeinsame Repräsentation vom Gegenüber" im Sinne eines Introjekts.

P. Eine schon vor dem Übergang ins VS bestehende, tragfähige Beziehung wird im VS als förderlich erlebt. Eine solche Beziehung konnte im VS mitunter vertieft werden. Das VS gestattet außerdem neue Beziehungserfahrungen, die als therapiefördernd erlebt werden, wie etwa das Erleben des Zusammenhalts in der Beziehung. Auch die Patient*innen selbst nehmen einen Zuwachs ihrer Kontrolle über die Interaktion durch das VS wahr. Wenn technische Probleme gemeinsam gelöst werden, hat dies eine Stärkung der therapeutischen Allianz zur Folge. Mehrheitlich sind die befragten Patient*innen für die Möglichkeit, das VS im Sinne einer Aufrechterhaltung der therapeutischen Beziehung nutzen zu können, dankbar.

\section{Therapeutischer Prozess}

T. Der therapeutische Prozess ist im VS weniger flüssig und dadurch erschwert. Patient*innen nutzten das VS mitunter, um etwas auszuagieren oder zu vermeiden. Ängstliche oder vermeidende Patient*innen können sich im VS, laut $\mathrm{PiA}$, leichter öffnen, wodurch neue Themen aufbrechen und der therapeutische Prozess angeregt wird.

P. Der therapeutische Prozess wird im VS als flacher und weniger intensiv erlebt, was den therapeutischen Fortschritt hemmt. Patient*innen bekommen weniger von ihren Therapeut*innen mit. Im Anschluss an die Sitzungen im VS fällt es ihnen schwerer, innerlich mit der Sitzung abzuschließen. Es ist außerdem eine Gewöhnungszeit an das VS notwendig. Im VS ist es v. a. ängstlichen Patient*innen möglich, ihre Abwehr partiell zu überwinden, was dazu führt, 
dass sie sich sowohl inhaltlich als auch emotional besser öffnen können.

\section{Therapeutische Technik}

T. Im VS wird die Übertragung schwächer, seltener und dabei anstrengender. Zudem wird ein verstärktes Auftreten von Abwehrmechanismen beobachtet. Stabilisierende, strukturstärkende Arbeit sowie Eye Movement Desensitization and Reprocessing (EMDR) sind im VS möglich. Erlebnisorientierte Techniken werden jedoch seltener umgesetzt. Die therapeutische Ich-Spaltung gelingt im VS weniger gut, und die Arbeit an Konflikten sowie die "Leerer-Stuhl-Technik" sind im VS schwer umsetzbar. Achtsamkeitsübungen, imaginative Techniken und weitere Techniken, die die Fantasie verstärkt ansprechen, sind im VS ebenso weniger möglich. Analytische Techniken wie Traumdeutung, freies Assoziieren und die aufdeckende Arbeit sind im VS nur eingeschränkt möglich. Dies trifft auch auf das emotionale "Mitschwingen" sowie auf die Funktionen "holding" und "containing" zu. Im VS wird tendenziell schneller interveniert als im PS.

P. Im VS kommentieren Therapeut*innen den therapeutischen Prozess verstärkt. Hierbei wird speziell auf die Körpersprache der Patient*innen eingegangen. Falls diese im Rahmen des VS nicht sichtbar ist, wird sie zudem erfragt.

\section{Themen}

T. Die intersubjektive Ebene der therapeutischen Beziehung fließt im VS plötzlich verstärkt in die besprochenen Themen ein, ebenso die Abwesenheit von der Praxis, die Abwehrstruktur sowie spezifische Widerstände. Mit dem VS verbundene Fragen der therapeutischen Technik, des persönlichen Kompetenzerlebens sowie die bestehende Vertrautheit unter den neuen Umständen werden innerhalb der Sitzungen zum Thema. Als die zentralsten Themen stellen sich jedoch COVID-19, Quarantäne, existenzielle Krisen sowie Ängste im Zusammenhang mit der Pandemie heraus. Schambesetzte Themen, wie die Sexualität, können nach Einschätzung der PiA im VS leichter angesprochen werden als im PS.
P.Im VS werden einige Themen oberflächlicher behandelt. Nach einem Verbindungsabbruch nimmt die Hemmung zu. Einige Themen sind deshalb nur im PS zu thematisieren.

\section{Kernkategorie}

Jeder Setting-Wechsel ist mit einem Habituationsprozess verbunden. Erst nach einigen Sitzungen kann die therapeutische Arbeit daher wieder mit der höchst möglichen Intensität, innerhalb des jeweiligen Settings, durchgeführt werden.

\section{Erleben des Setting-Wechsels}

T. Mit Vorerfahrung im VS fällt der Wechsel leichter. Die Entscheidung, ob sich die Patient*innen auf das VS einlassen oder nicht, hängt von der Einstellung der Therapeut*innen ab. Jeder Setting-Wechsel, sowohl ins VS als auch wieder zurück ins PS, erfordert eine Gewöhnungsphase. Im Laufe der Zeit ist es jedoch möglich, flexibler mit dem Setting-Wechsel umzugehen. Nach dem Wechsel zurück ins PS werden Patient*innen intensiver wahrgenommen.

P. Der Setting-Wechsel wird von stressbehaftet und ungewohnt bis hin zu problemlos erlebt. Mit dem neuen Setting konfrontiert, wird das VS, insbesondere von Patient*innen mit Angsterkrankungen, sogar bevorzugt. Sind Therapeut*innen noch unerfahren im VS, wird dies z.T. von den Patient*innen bemerkt.

\section{Allgemeine Erfahrungen mit dem Videosetting}

T. Im VS fehlt die „Natürlichkeit“ des PS. Die Psychotherapie beinhaltet im VS vermehrt edukative Elemente, sodass sie eher einer Beratung oder einem Coaching gleicht. Behandlungen werden als anstrengender erlebt, weshalb sich häufig das Gefühl, gestresst oder genervt zu sein, einstellt. Dem schließt sich überdies die Vermutung einer verringerten Wirksamkeit der Therapie im VS an.

P. Hinsichtlich seiner Wirksamkeit wird das VS im Vergleich zum PS als ambivalent erlebt. Das VS wird außerdem als eigenständige Therapieform wahrgenommen. Insgesamt wird das VS als angenehm be- schrieben, obwohl einige Patient*innen von verstärkten Ablenkungen innerhalb ihres privaten Umfelds berichten.

\section{Diskussion}

Interpretation der Ergebnisse und zukünftig zu falsifizierende Erkenntnisse

Im Rahmen der vorliegenden Studie wurde untersucht, wie der Wechsel vom PS ins VS (und wieder zurück) von Patient*innen und Therapeut ${ }^{*}$ innen erlebt wurde. Es zeigte sich, dass alle Studienteilnehmer*innen von der VBT profitiert haben. Besonders hervorgehoben wurde die vom VS ausgehende Flexibilität. Neben einigen in erster Linie praktischen Vorteilen wurden jedoch auch Nachteile, etwa bezüglich der empfundenen, eingeschränkten Wirksamkeit der Behandlung im VS benannt. Eine erhöhte Variabilität in der Gestaltung des Settings wurde außerdem sowohl von Patient*innen als auch von Therapeut*innen als verunsichernd beschrieben. Die therapeutische Situation wird im VS als durchlässiger und weniger berechenbar erlebt. Es besteht die Gefahr einer Entgrenzung des professionellen psychotherapeutischen Raums (Eichenberg 2020). Das zeigte sich z. B. darin, dass manche Psychotherapeut*innen angaben, die VBT auch von zu Hause aus durchzuführen. Dies widerspricht der Vorgabe der Bundespsychotherapeutenkammer (BPtK), die VBT nur innerhalb der eigenen Praxisräume, d.h. dem zugelassenen Dienstsitz, durchzuführen (Bundespsychotherapeutenkammer 2020). Gleichzeitig ergeben sich im VS jedoch auch Chancen für den psychotherapeutischen Prozess.

Zunächst erfüllt die VBT in Zeiten der COVID-19-Pandemie die Funktion einer Erhaltungstherapie, indem sie dem Kontakt zwischen Patient*in und Therapeut*in Kontinuität verleiht. Gleichzeitig flachen die Interaktionen im therapeutischen Prozess ab. Übertragung und Gegenübertragung werden als verändert erlebt, wofür das Fehlen wichtiger Wahrnehmungsinhalte entscheidend zu sein scheint (Roesler 2017). Diese Deprivation könnte eine weniger gerichtete therapeutische Interaktion zur Folge haben, die nach Roesler (2017) auf projektive Prozes- 
se, durch die die fehlenden Informationen ersetzt werden sollen, zurückgeführt werden kann. Weiterhin könnte angenommen werden, dass innerhalb des VS auf beiden Seiten eine vergleichsweise verstärkte kognitive Wahrnehmungs- und Verarbeitungsebene aktiviert wird (Simpson et al. 2021), während im PS unter Einwirkung der physischen (vs. virtuellen) Präsenz des Gegenübers eine sinnlichere Ebene und damit ein ganzheitlicheres emotionales Erleben möglich werden (Roesler 2017). Dieser Zusammenhang könnte mitunter dazu beigetragen haben, dass einige Studienteilnehmer*innen von einem abgeschwächten Übertragungsgeschehen berichtet haben. Dies ist v.a. im Hinblick auf Behandlungen im Bereich der AP von Bedeutung, da Übertragung und Gegenübertragung hier eine zentrale Rolle spielen. Das Angebot der VBT, in Verbindung mit der gemeinsamen Überwindung von Hindernissen, scheint sich jedoch insgesamt positiv auf die therapeutische Allianz auszuwirken. Einige Themen, wie z.B. die Sexualität, konnten im VS sogar besser thematisiert werden. Es kann in einigen Fällen also von einem „intensification loop" ausgegangen werden (Eichenberg 2011). Auch dies ist anhand der in den Hintergrund tretenden emotionalen Ebene erklärbar. Es kann angenommen werden, dass im VS zuvor schwerer thematisierbare Inhalte aufgrund der abgeschwächten emotionalen Intensität, in Verbindung mit der Betonung einer kognitiven, sachlichen Ebene, zumindest inhaltlich leichter zu besprechen, wenn auch schwerer erlebbar sind. Gleichzeitig könnte dies erklären, weshalb beispielsweise das freie Assoziieren, das das Verlassen einer kontrolliert kognitiven Ebene voraussetzt, im VS vergleichsweise schwerer fiel. Auch liefert dieser Ansatz eine Erklärung für die Bevorzugung des VS bei vermeidenden oder ängstlichen Patient*innen („online calming hypothesis"; Reynolds et al. 2013).

Dem folgend lässt sich das VS auch als Übergangsraum verstehen, der den Patient ${ }^{*}$ innen einen flexibleren Umgang mit dem Selbst und der eigenen Identität ermöglicht (Roesler 2017). Der*die Patient*in bekommt durch das VS mehr Kontrolle und Gestaltungsmöglichkeiten. So können z.B. Audio- und Videorepräsentationen an si- tuative Bedürfnisse angeglichen werden, wie etwa durch das Ausschalten der Kamera in Momenten überfordernder emotionaler Nähe. Das Auftreten von Stress und Unsicherheit kann als Teil eines Habituationsprozesses an einen neuen Möglichkeitsraum verstanden werden, der einerseits depriviert und andererseits Zugang zu neuen Möglichkeiten gewährt. Dennoch wird die Wirksamkeit des VS sowohl von den Therapeut*innen als auch den Patient*innen als insgesamt geringer eingeschätzt. Dem stehen in begrenztem Umfang Beobachtungen gegenüber, die zunächst eine Nichtunterlegenheit der VBT nahelegen (Backhaus et al. 2012; Berryhill et al. 2019; Norwood et al. 2018). Im psychodynamischen Bereich liegen aktuell jedoch lediglich Einzelfallberichte bezüglich der Wirksamkeit der VBT vor (Eichenberg und Hübner 2018). Psychodynamisch arbeitende Therapeut*innen sind, auch aufgrund des Mangels an vorliegenden schulenspezifischen Wirksamkeitsnachweisen, vermutlich eher skeptisch in Bezug auf die zu erwartende Wirksamkeit der VBT bei ihren Patient*innen. Es erscheint angesichts der beschriebenen Veränderungen, welche im Zuge eines Wechsels zwischen den verschiedenen Therapiesettings (VS, PS) zum Tragen kommen, jedoch sinnvoll, zu hinterfragen, inwiefern vereinzelt intermittierende Sitzungen im VS für den Therapieprozess bereichernd sein könnten.

Nachfolgend werden einige Hypothesen dargestellt, die innerhalb des Forschungsteams durch die Arbeit am Material entstanden sind. Im weitesten Sinne erfassen sie die vorläufigen Erkenntnisse, die in zukünftigen Studien noch zu falsifizieren sind:

1. Die Dauer der therapeutischen Beziehung im PS hat einen Einfluss auf die Intensität und Tiefe des therapeutischen Prozesses im VS, wobei eine bereits länger bestehende Therapie einen positiveren Prozess erwarten lässt.

2. Der Umgang mit technischen Problemen im VS erfordert beidseitig eine höhere Ich-Leistung, da die Fähigkeit zur therapeutischen Ich-Spaltung relevant ist.

3. Die Phänomenologie der Erkrankungen ändert sich nach einem Wechsel zum VS. Die Erkrankungen präsen- tieren sich in Abhängigkeit von der Struktur der Patient*innen anders als im PS.

4. Insgesamt kommt es im Rahmen des VS zu einer Regression hin zu ontogenetisch früher durchlaufenen Themen wie Nähe, Kontrolle und Bindung.

5. Das Phänomen der Übertragung verändert sich im VS, wodurch die Arbeit mit der Übertragung, zum einen als Diagnostikum, zum anderen als Basis deutender Interventionen, erschwert werden kann. Gleichzeitig sind im VS andere Übertragungsangebote vorhanden, wie z. B. im Hinblick auf den privaten Raum, der, wenn der*die Therapeut*in das VS von zu Hause aus durchführt, von beiden Seiten mehr oder weniger in den Prozess einfließen kann. Auch der Umgang mit technischen Störungen, wobei der*die Therapeut*in durchaus hilflos erlebt werden kann, könnte bezüglich des Übertragungsgeschehens von Bedeutung sein.

6. Für vermeidende Patient*innen wirkt das VS insofern erleichternd, als angstbesetzte Themen angesprochen werden bzw. überhaupt eine Therapie initiiert wird, da der Weg zur Therapiepraxis entfällt.

7. Die Kontrollüberzeugung der Patient ${ }^{*}$ innen (Hovenkamp-Hermelink et al. 2019) verschiebt sich im VS zu ihren Gunsten. Einerseits wird so die Bearbeitung der sonst vermiedenen Themen möglich, andererseits kann es bei Angstpatient*innen dazu führen, dass die Begegnung mit dem*der Therapeut*in vermieden wird. Demnach kommt es nach einem Wechsel ins VS auch zu einer Veränderung in der Abwehr der Patient*innen.

\section{Methodische Limitationen und Forschungsausblick}

Im Zuge der Rekrutierung wurde im Sinne einer entsprechenden Vorauswahl bezüglich der Stichprobenzusammensetzung, gemäß einem qualitativen Forschungsansatz, wenig Einfluss genommen, sodass die befragten Personen nicht repräsentativ für die Gruppe der Therapeut*innen oder Patient*innen ausgewählt wurden. 
Weiterhin wurde durch das angewandte Convenience sampling eine weitere Differenzierung zwischen den selektiven Kodes gehemmt, wodurch auch die theoretische Gültigkeit der vorgestellten Annahmen entsprechend limitiert ist. Die vorgestellten Thesen sollen vielmehr zur Diskussion anregen und zu weiterer Forschung innerhalb dieses Themenfelds inspirieren. Eine weitere Schwachstelle ist, dass zwischen den psychodynamischen Verfahren (AP, TP) oder zwischen dem Ausmaß gemachter Erfahrung mit dem VS nicht systematisch unterschieden wurde. Im Sinne einer zusätzlichen Absicherung der Ergebnisse hätten diese den Studienteilnehmer*innen im Anschluss an die Auswertung zur Evaluierung außerdem vorgelegt werden können. Weiterhin wurden die gemachten Erfahrungen retrospektiv erfragt, und in einigen Fällen lag eine größere Zeitspanne zwischen Setting-Wechsel und Interview. Außerdem könnte es eine Rolle spielen, zu welchem Zeitpunkt der Pandemie und innerhalb des therapeutischen Verlaufs ein Wechsel stattfand und zu wie vielen SettingWechseln es insgesamt kam, was im Rahmen dieser Untersuchung ebenfalls nicht gezielt berücksichtigt wurde. Auch wurde die Dauer der Behandlungen im VS nicht erhoben, was im Hinblick auf den beschriebenen Habituationsprozess jedoch von erheblicher Bedeutung sein könnte. Eine Habituierung an das neue Therapie-Setting kann möglicherweise nicht bei allen Therapieverfahren innerhalb der als Einschlusskriterium vorgegebenen Mindestanzahl von 5 Sitzungen im VS erreicht werden. Aus diesem Grund könnten bestimmte Techniken, wie das freie Assoziieren oder die Traumdeutung, als im VS eher ungeeignet beschrieben worden sein. Dies würde im Hinblick auf die AP beispielsweise gegen einen häufigeren Setting-Wechsel sprechen, da die immer wieder stattfindenden Habituationsprozesse die Arbeit in der Regression behindern würden. Dem gegenübergestellt ist innerhalb der TP eine geringere Habituationsdauer vorstellbar. Vor diesem Hintergrund scheint es wichtig, diese Prozesse für AP und TP in weiteren Studien getrennt zu beleuchten. Auch bezüglich eines Setting-spezifischen Übertragungsangebots (s. oben: Interpretation der
Ergebnisse und zukünftig zu falsifizierende Erkenntnisse), wäre es perspektivisch sinnvoll, länger andauernde Therapieprozesse im VS zu untersuchen, da sich diese Veränderungen möglicherweise erst im Laufe des Prozesses ergeben. Weitere Studiendesigns sollten außerdem so konzipiert sein, dass auch Therapeut*innenPatient*innen-Paare befragt werden, wodurch es möglich wäre, Abstimmungsprozesse sowie bestimmte Übertragungsund Gegenübertragungskonstellationen innerhalb der therapeutischen Dyade zu untersuchen.

Vor dem Hintergrund der beschriebenen Einschränkungen dieser Studie sind weitere Bemühungen zur Theoriebildung im Zusammenhang mit quantitativen Untersuchungen notwendig. Nur so kann eine empirisch fundierte Theorie zum psychodynamischen Prozess im VS entstehen. Die gebildeten Hypothesen können letztendlich nur die Grundlage für weiterführende und v.a. gerichtetere Untersuchungen bilden. Abschließend ist zudem festzuhalten, dass dem untersuchten abrupten Wechsel ins VS das Einsetzen der Pandemie zugrunde liegt. Die hiervon ausgehende Belastung, der alle an dieser Untersuchung beteiligten Personen ausgesetzt waren, führt zu einer möglichen Einschränkung der Generalisierbarkeit der formulierten Hypothesen auf nichtpandemische Bedingungen. Aus den vorliegenden Beobachtungen lassen sich weiterhin vorläufige Empfehlungen, einerseits für die Entwickler*innen zertifizierter Programme für die VBT und andererseits für die Therapeut*innen in der Praxis, ableiten.

\section{Fazit für die Praxis}

- Programmseitig ist es notwendig, Voraussetzungen für einen weitestgehend unmittelbaren Kontakt zu schaffen. Gestik und Mimik sollten mit kleinstmöglichen Latenzen übertragen werden, da dies für den Prozess der Affektabstimmung elementar ist. Therapeut*innen sollten die zertifizierten Programme - nicht nur aufgrund der rechtlichen Vorschriften - ausschließlich von ihrem Dienstsitz aus nutzen, da dies den Patient*innen Sicherheit vermittelt.

- Patient*innenseits ist zentral, den Umgang mit technischen Störungen explizit zu besprechen, da die Nichtbewältigung zu einer größeren Verunsicherung, die Bewältigung hingehen zu einer Stärkung der therapeutischen Beziehung führt. Zudem sollte ein im Vergleich zur Wegstrecke zwischen zu Hause und Therapiepraxis alternativer Übergang zwischen Alltag und Therapie gefunden werden, der in der Therapie thematisiert wird.

- Therapeut*innenseits wäre ein empirisch fundiertes Manual zur Durchführung psychodynamisch fundierter videobasierter Therapien (VBT) ein enormer Zugewinn. Bisherige allgemeine Manuale zur VBT enthalten kaum Hinweise zur Behandlungstechnik.

Korrespondenzadresse

Alena Leukhardt

Department Psychotherapiewissenschaft, Sigmund Freud PrivatUniversität Berlin Columbiadamm 10, 12101 Berlin, Deutschland alena.leukhardt@sfu-berlin.de

\section{Einhaltung ethischer Richtlinien}

Interessenkonflikt. A. Leukhardt, M. Heider, K. Reboly, G. Franzen und C. Eichenberg geben an, dass kein Interessenkonflikt besteht.

Alle beschriebenen Untersuchungen am Menschen wurden mit Zustimmung der Ethikkommission der Sigmund Freud PrivatUniversität (Nummer des Ethikvotums: CBQDRZLBAPCQYK87996), im Einklang mit nationalem Recht sowie gemäß der Deklaration von Helsinki von 1975 (in der aktuellen, überarbeiteten Fassung), durchgeführt. Von allen befragten Patient*innen und Therapeut*innen liegt eine Einverständniserklärung vor.

\section{Literatur}

Backhaus A et al (2012) Videoconferencing psychotherapy: a systematic review. Psychol Serv 9(2):111-131. https://doi.org/10.1037/ a0027924

Barber JP, Walczak KK (2009) Conscience and critic: peer debriefing strategies in grounded theory research. In: Annual meeting of the American Educational Research Association San Diego

Békés V, Aafjes-van Doorn K (2020) Psychotherapists' attitudes toward online therapy during the COVID-19 pandemic. J Psychother Integr 30(2):238-247. https://doi.org/10.1037/ int0000214

Berryhill MB et al (2019) Videoconferencing psychological therapy and anxiety: a systematic review. Fam Pract 36(1):53-63. https://doi.org/10.1093/ fampra/cmy072

Breuer F, Muckel P, Dieris B (2019) Reflexive grounded theory. Springer, Wiesbaden https://doi.org/10. 1007/978-3-658-22219-2

Bundespsychotherapeutenkammer (2020) Praxisinfo Videobehandlung. https://www.bptk.de/wpcontent/uploads/2020/03/bptk_praxisInfo videobehandlung_21_web.pdf. Zugegriffen: 2 . Juli 2021

Corbin J, Strauss A (2015) Basics of qualitative research. Techniques and procedures for developing grounded theory. SAGE, USA 
Deutsche Psychotherapeuten Vereinigung (2020) Umfrage Psychotherapeutische Videobehandlung. https://www.

deutschepsychotherapeutenvereinigung.de/ index.php?elD $=$ dumpFile $\& \mathrm{t}=\mathrm{f} \& \mathrm{f}=11152 \&$ token $=$

8efba22d7afdbd29ab5f0a824eb29c7d2aa94b9c. Zugegriffen: 15. Mai 2020

Dourdouma A, Mörtl K (2012) The creative journey of grounded theory analysis: a guide to its principles and applications. Res Psychother 15(2):96-106. https://doi.org/10.4081/ripppo. 2012.108

Eichenberg C (2011) Psychotherapie und Internet. Psychotherapeut 56(6):468-474. https://doi. org/10.1007/s00278-011-0865-9

Eichenberg C (2020) Psychotherapieim Online-Setting: Varianten, Besonderheiten und potenzielle Grenzverletzungen. Imagination 4:17-29

Eichenberg C (2021) Onlinepsychotherapie in Zeiten der Coronapandemie. Psychotherapeut. https:// doi.org/10.1007/s00278-020-00484-0

Eichenberg C, Hübner L (2018) Psychoanalyse via Internet: Aktueller Stand der Diskussion um Möglichkeiten und Grenzen. Psychotherapeut 63(4):283-290. https://doi.org/10.1007/ s00278-018-0294-0

Glaser BG, Strauss AL (1967) The discovery of grounded theory. Strategies for qualitative research. Aldine Publishing Company, Chicago

Hartmann-Strauss S (2020) Videotherapie und Videosupervision: Praxishandbuch Für Psychotherapie und Beratung Online. Springer, Berlin, Heidelberg

Hoffmann M et al (2020) Integration if mental health care within primary care via video consultation: a qualitative pre-implementation study exploring the perspectives of psychotherapists and psychiatrists. J Med Internet Res 22(6):e17569. https://doi.org/10.2196/17569

Hovenkamp-Hermelink JH et al (2019) Differential associations of locus of control with anxiety, depression and life-events: a five-wave, nineyear study to test stability and change. J Affect Disord 253:26-34. https://doi.org/10.1016/j.jad. 2019.04.005

Jaeger B, Furck C (2014) Neue Medien und Psychoanalyse - Fluch und Chance. In: Wahl P, Lehmkuhl U (Hrsg) Seelische Wirklichkeiten in virtuellen Welten. Vandenhoeck \& Ruprecht, Göttingen, S225-244

Norwood C, Moghaddam NG, Malins S, Sabin-Farrell R (2018) Working alliance and outcome effectiveness in videoconferencing psychotherapy: a systematic review and noninferiority metaanalysis. Clin Psychol Psychother 25(6):797-808. https://doi.org/10.1002/cpp.2315

Reese RJ et al (2016) The effects of telepsychology format on empathic accuracy and the therapeutic alliance: an analogue counselling session. Couns Psychother Res 16(4):256-265. https://doi.org/ 10.1002/capr.12092

Reynolds DAJ Jr, Stiles WB, Bailer AJ, Hughes MR (2013) Impact of exchanges and client-therapist alliance in online-text psychotherapy. Cyberpsychol Behav Soc Netw 16(5):370-377. https://doi.org/ 10.1089/cyber.2012.0195

Roesler C (2017) Tele-analysis: the use of media technology in psychotherapy an its impact on the therapeutic relationship. J Anal Psychol 62(3):372-394. https://doi.org/10.1111/14685922.12317

Scharff JS (2013) Psychoanalysis online: mental health, teletherapy, and training. Karnac Books, London
Video-based treatment in psychodynamic psychotherapy in times of the COVID-19 pandemic. Interview study with patients and psychotherapists

Background: In the course of the pandemic triggered by the coronavirus disease 2019 (COVID-19), video-based treatment (VBT) has undergone a trend reversal. It must be assumed that the abrupt switch from traditional psychotherapy to the video setting (VS) has an impact on the therapeutic relationship and thus on the therapeutic process. This study examined how the switch from traditional treatment to VBT (and back again) during the COVID-19 pandemic was experienced by patients and therapists with respect to the therapeutic relationship and the therapeutic process.

Methods: Group-specific, semi-structured interviews with therapists $(n=8)$, therapists in training $(n=6)$, and patients $(n=9)$.

Results: The results show that the majority of participants, both therapists as well as patients, experienced the therapeutic situation in VS as more permeable and less predictable. Although the VS contributes to maintenance of the therapeutic situation, the interaction was described as shallow and less oriented. Furthermore, every setting change was associated with a process of habituation. Therefore, the therapeutic work can only be carried out with the highest possible intensity after several sessions within each setting.

Conclusion: The possibility of maintaining the therapeutic relationship using VBT can lead to a strengthening of the therapeutic alliance. Although the VS is associated with uncertainties, shame-ridden topics can be addressed more freely. Anxiety patients, in particular, could engage in a more intense therapeutic process after a universally observed phase of habituation.

\section{Keywords}

Setting change - Therapeutic process - Professional-patient relation - Patient-therapist interaction . Online therapy

Simpson S, Richardson L, Pietrabissa G, Castelnuovo G Reid C (2021) Videotherapy and therapeutic alliance in the age of COVID-19. Clin Psychol Psychother 28(2):409-421. https://doi.org/10. 1002/cpp.2521

Software VERBI (2019) MAXQDA 2020 [computer software]. VERBISoftware, Berlin

Tonn P et al (2017) Development of a questionnaire to measure the attitudes of laypeople, physicians, and psychotherapists toward telemedicine in mental health. JMIR Ment Health 4(4):e93. https://doi.org/10.2196/mental.6802

White K (2020) Practicing as an analyst in Berlin in times of the coronavirus: the core components of psychoanalytic work and the problem of virtual reality. Int J Psychoanal 101(3):580-584. https:// doi.org/10.1080/00207578.2020.1761816 